\title{
Reliability and validity of time domain heart rate variability during daily routine activities - an alternative to the morning orthostatic test?
}

\author{
Ricardo Neves de Oliveira Mesquita ${ }^{1}$, Heikki Kyröläinen ${ }^{1}$, Daniela Schäfer Olstad² \\ ${ }^{1}$ Department of Biology of Physical Activity, University of Jyväskylä, Finland; ${ }^{2}$ Polar Electro Oy, Kempele, Finland
}

\section{Summary}

Study aim: To determine the reliability and validity of a time domain heart rate variability (HRV) index during free-living physical activity (FLPA).

Material and methods: Eight white-collar workers participated in this study. RR intervals (time between consecutive R-peaks of the PQRS complex) were recorded using the Polar V800 heart rate (HR) monitor upon awakening and at work on 16 different days. A total of 127 cycles of sitting periods followed by walking breaks were included for consecutive pairwise analysis for reliability. The HR values from the orthostatic test (OT) were compared with the corresponding values at work.

Results: The HR values showed high levels of repeatability [the coefficient of variation (CV) during sitting and walking at work was 4.71 and $3.99 \%$, respectively, with a typical error (TE) of 3.73 (3.34-4.25) and $3.65(3.31-4.09)]$, but they did not correlate with the corresponding OT HR upon awakening $(r=0.28$ for supine vs. sitting and $r=0.05$ for standing vs. walking, $p>0.05)$. The root-mean-square difference of successive normal RR (RMSSD) was revealed not to be repeatable [CV values during sitting and walking were 19.99 and $29.05 \%$, respectively, with a TE of 7.9 (7.15-8.85) and $9.43(8.53-10.57)]$.

Conclusions: Analyzing RMSSD from daily routine activities was not reliable, and therefore validity cannot be assumed. RMSSD should therefore be calculated from RR intervals recorded in standardized conditions, such as during the OT upon awakening.

Keywords: Recovery - RMSSD - Monitoring - Validity - Reliability

\section{Introduction}

Balancing training and recovery is one of the biggest challenges among recreationally active people, coaches and athletes. It is paramount to monitor training responses and other life stressors in order to avoid insufficient training load or too high training load that could lead to nonfunctional overreaching or overtraining [17].

Measuring heart rate variability (HRV) constitutes an interesting, non-invasive and simple tool to monitor training and physiological responses [4]. Through this method, possible changes in the cardiac autonomic nervous system (ANS) induced by training may be inferred while using repeated HRV measures over time. To date, several studies have demonstrated a high sensitivity of HRV to physical fitness improvement, fatigue, overload or detraining $[3,12,15,16,22,27]$, which supports the idea that HRV may be used to tailor training. The general idea is based on the assumption that athletes should only train at a high intensity level when HRV indices have returned to baseline values [7, 29]. The root-mean-square difference of successive normal RR (RMSSD), which reflects cardiac parasympathetic activity, has been demonstrated to be a promising tool to monitor recovery status over short [27] and long [24] training periods. Changes in this time domain index may provide crucial information, when concurrently taking training context and changes in HR into consideration [7].

The orthostatic test (OT), where RR intervals are measured for 5 minutes in a supine position followed by 5 minutes in a standing position, is widely used to measure HRV indices [26]. Some recent studies [10, 18, 21] have shown that a shorter segment length can be used as an alternative. Still, in an ideal scenario, the feasibility in daily use would improve considerably if HRV indices could be analyzed during free living physical activity (FLPA). Thus, the aim of this study was to determine the reliability and validity of a time-domain HRV index during daily routine situations. To the best of our knowledge, this is the 
first scientific study to address the possibility of measuring HRV during FLPA.

\section{Material and methods}

\section{Participants}

Eight (5 men and 3 women) white-collar workers ( $35 \pm 11$ years, $175.2 \pm 9.79 \mathrm{~cm}, 78.84 \pm 10.45 \mathrm{~kg}$ ), who recreationally engage in physical exercise, were recruited to participate in this study. Participants were not taking cardioactive medications and did not have any cardiovascular problems that may have influenced the findings of the present study. The procedures were approved by the local Ethics Committee.

\section{Study design}

The HRV data were collected with a personal HR monitor (V800, Polar Electro Oy, Kempele, Finland) every morning at home upon awakening and at work during routine situations on 16 different days. In the morning protocol of day 1, RR intervals were measured at quiet rest in 3 minutes of supine and 3 minutes of standing postures. After this 6-minute period, participants lay down again for 5 minutes and then walked for 30 seconds. In the morning protocol of day 2, participants first lay down for 3 minutes and then got up and walked for 30 seconds. Thereafter, participants remained in a supine position for another 8 minutes followed by a standing position for 3 minutes. Morning procedures on day 3 were the same as on day 1, and morning procedures on day 4 were the same as day 2 , and this pattern was repeated until day 16. At work, participants were asked to turn on the RR-recording feature when taking a sitting position for the first time. They were then asked to report the time when they got up to walk around and when they sat down again. Participants stopped the $\mathrm{RR}$ recording after repeating this process 3 times. These procedures were defined in order to enable a comparison between supine and sitting positions that should mainly reflect parasympathetic modulation [25], whereas a reduction in vagal tone is expected during standing and walking after longer resting periods [25].

\section{HRV analysis}

RR interval data were exported to HRV analysis software (Kubios HRV Analysis, version 2.2, The Biomedical Signals Analysis Group, University of Kuopio, Finland). In the mornings of days 1 and 3 , the final 2 minutes of recording in the supine and standing positions were analyzed, as well as the 30 -second walking period. In the mornings of days 2 and 4, the 30-second walking period was analyzed, as well as the final 2 minutes of the supine and standing positions performed at the end of the protocol. At work, the last 2-minute period of each sitting period was analyzed as well as the first 30 seconds of the walking bouts. Mean HR and RMSSD were analyzed. Artefacts were visually inspected and corrected with automated correction filters from the Kubios HRV Analysis software.

\section{Statistical analysis}

Consecutive pairwise analysis of trials for reliability [typical error (TE) with upper and lower confidence limits, coefficient of variation $(\mathrm{CV})$ and intraclass correlation coefficient (ICC) were calculated] was performed using a published spreadsheet [14] with the $95 \%$ confidence limit. As HR was shown to be reliable, its mean values measured during OT in the morning were correlated with the corresponding values during daily routines (i.e. first supine period vs. sitting at work, standing vs. walking at work) for each participant, where the mean values within each cycle of sitting and walking at work were used. The RMSSD values from the standing position were also associated with the respective values from the walking period upon awakening. Pearson product-moment correlation was used when data were normally distributed and Spearman's rank order correlation for non-normally distributed data.

\section{Results}

A total of 127 cycles of sitting periods followed by walking breaks were included for consecutive pairwise analysis of trials for reliability. On average, participants completed the measurements at work 152.60 (130.57) minutes after the measurements upon awakening. At work, participants took 68.60 (43.20) minutes to complete the repeated sitting and walking bouts.

Values of reliability can be seen in Table 1. During the morning OT, the mean HR and RMSSD values demonstrated an excellent and moderate level of reliability, respectively. Furthermore, mean HR during sitting and walking at work showed high levels of repeatability. However, RMSSD was not repeatable in the studied daily routine situations. HR data recorded upon awakening did not correlate with the corresponding values at work $(\mathrm{r}=0.28$ for supine vs. sitting and $\mathrm{r}=0.05$ for standing vs. walking, $\mathrm{p}>0.05)$. Only one of the participants showed a moderate positive correlation between standing position upon awakening and walking at work, which was statistically significant $(r=0.506, p<0.05)$. Furthermore, the RMSSD values analyzed from the recordings during standing did not correlate with the respective values during walking in the morning upon awakening $(\mathrm{r}=0.138, \mathrm{p}>0.05)$, with the exception of one participant $(r=0.591, p<0.05)$. 
Table 1. Consecutive pairwise analysis of trials for reliability. Typical Error (TE), Coefficient of Variation (CV) and Intraclass Correlation Coefficient (ICC) of mean HR and RMSSD during sitting and walking at work

\begin{tabular}{|c|c|c|c|}
\hline Situation & $\begin{array}{l}\text { Typical error (lower and upper } \\
\text { confidence limits) }\end{array}$ & Typical error as CV & $\begin{array}{c}\text { Intraclass correlation } \\
\text { coefficient }\end{array}$ \\
\hline Supine position & 1.86 & $3.5 \%$ & 0.96 \\
\hline (Mean HR) & $(1.57-2.26)$ & & \\
\hline Supine position & 9.7 & $12.3 \%$ & 0.89 \\
\hline (RMSSD) & $(8.22-11.83)$ & & \\
\hline Sitting at work & 3.72 & $4.7 \%$ & 0.84 \\
\hline (Mean HR) & $(3.23-4.45)$ & & \\
\hline Sitting at work & 7.88 & $20.0 \%$ & 0.79 \\
\hline (RMSSD) & $(7.16-8.88)$ & & \\
\hline Walking at work & 3.64 & $4.0 \%$ & 0.85 \\
\hline (Mean HR) & $(3.30-4.08)$ & & \\
\hline Walking at work & 9.43 & $29.1 \%$ & 0.47 \\
\hline (RMSSD) & $(8.57-10.42)$ & & \\
\hline
\end{tabular}

\section{Discussion}

To the best of our knowledge, this is the first scientific study to address the possibility of measuring HRV during FLPA. The present results demonstrate that the vagal-related HRV index used in this study (RMSSD) does not have high repeatability during FLPA at work in the mornings when calculated from the RR intervals measured during consecutive cycles of sitting periods followed by short walking working breaks. This information is of great importance for athletes, coaches and recreationally active people, as they would get a bias interpretation when assessing changes in this marker during FLPA. The CV reported in this study during FLPA (19.99 and 29.05\% for sitting and walking, respectively) is considerably higher compared to the CV of $12.27 \%$ found in the supine position of the OT in this study. Although our study design did not allow us to measure reliability of RMSSD in the standing position, high levels of reliability have previously been reported for this position [9]. It seems reasonable to infer that measuring HRV should be done in standardized conditions in order to obtain reliable and valid values that could be used to monitor training status. This low reliability during sitting and walking periods at work may be explained by external factors that can influence autonomic modulation, such as variability in breathing patterns, body position, noise, light, intensity of walking bouts, amount of light physical activity during the sitting periods and other work-related stressful events [1]. A recent study showed a high intra-day reliability ( $\mathrm{ICC}=0.96 ; \mathrm{CV}=3.99 \%$ ) of lnRMSSD assessments that were performed for 2 minutes in a standardized quiet sitting position [18]. Although intra-day reliability is highly meaningful, a note of caution is due here, as further research is needed to investigate whether measuring HRV in such standardized conditions would be as valid as morning measurements upon awakening. HRV parameters collected through recording RR intervals later in the morning at work or at a training facility may be influenced by the ANS circadian rhythm - a sudden rise of sympathetic activity and a withdrawal of vagal tone is expected during the morning hours [11]. For example, in our study, participants performed the RRinterval recording at work after $2 \mathrm{~h} 30 \mathrm{~min}$, approximately. Nevertheless, the low reliability of RMSSD during FLPA at work, which is the most meaningful research outcome of our study, can be explained by several factors previously discussed, and not by the ANS circadian rhythm, as the repeated sitting and walking situations at work were performed in a considerably short time window (around 69 minutes, on average).

Furthermore, it is important to mention that the validity of measuring HRV after active commuting (an important factor to take into consideration in further studies) should be questionable as post-exercise blood pressure regulation, baroreflex activity and metaboreflex stimulation could influence ANS [6, 27].

Although further research is needed with larger sample sizes in order to infer definitive conclusions, Plews et al. [23] demonstrated that HR may also be quite sensitive to monitor training status, as there was only a slightly stronger correlation between HRV and non-functional overreaching, when compared to HR ( $r=0.88$ vs 0.81$)$. In the present study, mean HR was repeatable during FLPA. 
However, a strong association with the respective values during OT was not found. Thus, the alternative of relying solely on mean HR during FLPA instead of HRV in standardized conditions should be discarded.

There is a wide range of methods to assess HRV [28], and they all have advantages and disadvantages. We have used RMSSD as it can be calculated from a short period of time [19], and it has been shown to be more suitable and feasible for monitoring athletes in the field [7]. Second$1 y$, these indices have a reduced sensitivity to respiration rates [20]. Furthermore, frequency domain indices could not be calculated during the short walking periods as they require a stable condition and a longer recording length [19]. In addition, it would be expected to find a higher CV for frequency-domain indices [2]. Sleep HRV recordings are another promising time-efficient alternative to morning HRV measures $[5,8,13]$, but sleep analysis was not the aim of this study.

\section{Practical applications}

It seems that recreationally active people and athletes should keep using standardized conditions to draw conclusions about their training status and make the best possible decisions regarding training load and recovery. It would be much more feasible to obtain this information from FLPA, but it does not appear to be a reliable procedure, and therefore its validity cannot be assumed.

\section{Conflict of interest: Authors state no conflict of interest.}

\section{References}

1. Achten J., Jeukendrup A.E. (2003) Heart rate monitoring: Applications and limitations. Sport Med., 33(7): 517-538.

2. Al Haddad H., Laursen P.B., Chollet D., Ahmaidi S., Buchheit M. (2011) Reliability of resting and postexercise heart rate measures. Int. J. Sports Med., 32(8): 598-605.

3. Bellenger C., Karavirta L. (2016) Contextualising parasympathetic hyperactivity in functionally overreached athletes with perceptions of training tolerance. Int. J. Sports Physiol. Perform., 11(7): 685-692.

4. Bosquet L., Merkari S., Arvisais D., Aubert A.E. (2008) Is heart rate a convenient tool to monitor over-reaching? A systematic review of the literature. Br. J. Sports Med., 42(9): 709-714.

5. Brandenberger G., Buchheit M., Ehrhart J., Simon C., Piquard F. (2005) Is slow wave sleep an appropriate recording condition for heart rate variability analysis? Auton. Neurosci., 121(1-2): 81-86.
6. Buchheit M., Papelier Y., Laursen P.B. (2007) Noninvasive assessment of cardiac parasympathetic function : postexercise heart rate recovery or heart rate variability? Am. J. Physiol. Heart Circ. Physiol., 293(1): 8-10.

7. Buchheit M. (2014) Monitoring training status with HR measures: Do all roads lead to Rome? Front. Physiol., 5(73): 1-19.

8. Buchheit M., Simon C., Piquard F., Ehrhart J., Brandenberger G. (2004) Effect of increased training load on vagal-related indexes of heart rate variability: a novel sleep approach. Am. J. Physiol. Heart Circ. Physiol., 287(6): H2813-H2818.

9. Dantas E.M., Gonçalves C.P., Silva A.B.T., Rodrigues S.L., Ramos M.S., Andreão R.V., Pimentel E.B., Lunz W., Mill J.G. (2010) Reproducibility of heart rate variability parameters measured in healthy subjects at rest and after a postural change maneuver. Brazilian. $J$. Med. Biol. Res., 43(10): 982-988.

10. Flatt A.A., Esco M.R. (2016) Evaluating individual training adaptation with smartphone-derived heart rate variability in a collegiate female soccer team. J. Strength Cond. Res., 30(2): 378-385.

11. Furlan R., Guzzetti S., Crivellaro W., Dassi S., Tinelli M., Baselli G., Cerutti S., Lombardi F., Pagani M., Malliani A. (1990) Continuous 24-hour assessment of the neural regulation of systemic arterial pressure and RR variabilities in ambulant subjects. Circulation. 81(2): 537-547. DOI:10.1161/01.CIR.81.2.537.

12. Garet M., Tournaire N., Roche F., Laurent R., Lacour J.R., Barthélémy J.C., Pichot V. (2004) Individual interdependence between nocturnal ANS activity and performance in swimmers. Med. Sci. Sports Exerc., 36(12): 2112-2118.

13. Herzig D., Testorelli M., Olstad D.S., et al. Heart rate variability during deep sleep offers a time-efficient alternative to morning supine measurements - a study in world class alpine skiers. Int. J. Sports Physiol. Perform., [Published online ahead of print September 2016: 1-22].

14. Hopkins W.G. (2015) Spreadsheets for analysis of validity and reliability. Sportsci., 19: 36-42.

15. Kiviniemi A.M., Tulppo M.P., Hautala A.J., Vanninen E., Uusitalo A.L. (2014) Altered relationship between R-R interval and R-R interval variability in endurance athletes with overtraining syndrome. Scand. J. Med. Sci. Sports, 24(2): e77-e85.

16. Le Meur Y., Pichon A., Schaal K., Schmitt L., Louis J., Gueneron J., Vidal P.P., Hausswirth C. (2013) Evidence of parasympathetic hyperactivity in functionally overreached athletes. Med. Sci. Sports Exerc., 45: 2061.

17. Meeusen R., Duclos M., Foster C., Fry A., Gleeson M., Nieman D., Raglin J., Rietjens G., Steinacker J., Urhausen A. (2013) Prevention, diagnosis, and treatment of the overtraining syndrome: Joint consensus statement of the European College of Sport Science and the Ameri- 
can College of Sports Medicine. Med. Sci. Sports Exerc., 45(1): 186-205.

18. Nakamura F.Y., Pereira L.A., Esco M.R., Flatt A.A., Moraes J.E., Cal Abad C.C., Loturco I. (2017) Intra- and inter-day reliability of ultra-short-term heart rate variability in rugby union players. J. Strength Cond. Res.. 31(2): 548-551.

19. Nussinovitch U., Elishkevitz K., Kaminer K., Nussinovitch M., Segev S., Volovitz B., Nussinovitch N. (2011) The efficiency of 10-second resting heart rate for the evaluation of short-term heart rate variability indices. Pacing Clin. Electrophysiol., 34(11): 1498-1502. DOI: 10.1111/j.1540-8159.2011.03178.x.

20. Penttilä J., Helminen A., Jartti T., Kuusela T., Huikuri H.V., Tulppo M.P., Coffeng R., Scheinin H. (2001) Time domain, geometrical and frequency domain analysis of cardiac vagal outflow: Effects of various respiratory patterns. Clin. Physiol., 21(3): 365-376.

21. Pereira L.A., Flatt A.A., Ramirez-Campillo R., Loturco I., Nakamura F.Y. (2016) Assessing shortened fieldbased heart-rate-variability-data acquisition in team-sport athletes. Int. J. Sports Physiol. Perform., 11(2): 154-158.

22. Pichot V., Roche F., Gaspoz J.M., Enjolras F., Antoniadis A., Minini P., Costes F., Busso T., Lacour J.R., Barthélémy J.C. (2000) Relation between heart rate variability and training load in middle-distance runners. Med. Sci. Sports Exerc., 32(10): 1729-1736.

23. Plews D.J., Laursen P.B., Kilding A.E., Buchheit M. (2012) Heart rate variability in elite triathletes, is variation in variability the key to effective training? A case comparison. Eur. J. Appl. Physiol., 112(12): 3729-3741.
24. Plews D.J., Laursen P.B., Stanley J., Kilding A.E., Buchheit M. (2013) Training adaptation and heart rate variability in elite endurance athletes: Opening the door to effective monitoring. Sport Med., 43(9): 773-781.

25. Pumprla J., Howorka K., Groves D., Chester M., Nolan J. (2002) Functional assessment of heart rate variability: physiological basis and practical applications. Int. J. Cardiol., 84(1): 1-14.

26. Schmitt L., Regnard J., Desmarets M., Mauny F., Mourot L., Fouillot J.P., Coulmy N., Millet G. (2013) Fatigue shifts and scatters heart rate variability in elite endurance athletes. (ed.): A. Zirlik, PLoS One, 8(8): e71588.

27. Stanley J., Peake J., Buchheit M. (2013) Cardiac parasympathetic reactivation following exercise: implications for training prescription. Sport Med., 43(12): 1259-1277.

28. Task Force. Heart rate variability: standards of measurement, physiological interpretation and clinical use. Task Force of the European Society of Cardiology and the North American Society of Pacing and Electrophysiology. Circulation. 1996, 93: 1043-1065.

29. Vesterinen V., Nummela A., Heikura I., Laine T., Hynynen E., Botella J., Häkkinen K. (2016) Individual endurance training prescription with heart rate variability. Med. Sci. Sports Exerc., 48(7): 1347-1354.

\section{Received 13.01.2017 \\ Accepted 19.04.2017}

(C) University of Physical Education, Warsaw, Poland 